\title{
Density of States of Twisted Bilayer Graphene at Low Energy
}

\author{
Muhamad Nasruddin Manaf ${ }^{1,2 a}$, Iman Santoso ${ }^{1,2, b}$ and Arief Hermanto ${ }^{1,2}$ \\ ${ }^{1}$ Jurusan Fisika, Fakultas Matematika dan Ilmu Pengetahuan Alam, Universitas Gadjah Mada, Bulaksumur 55281, \\ Yogyakarta, Indonesia \\ ${ }^{2}$ Yayasan Hikmah Teknosains, Jl. Kaliurang Km. 5,3 Gg. Pamungkas No. 16 A, Yogyakarta, Indonesia \\ amuhamad.nasruddin.manaf@mail.ugm.ac.id ; biman.santoso@ugm.ac.id
}

\begin{abstract}
We report the calculation of the Density of States (DOS) in Twisted Bilayer Graphene (TBG) system for low energy range near Fermi level using commensurate rotation of $1.16^{0}, 1.79^{0}$ and $3.48^{\circ}$. We obtain a logarithmic behaviour of DOS showing that van Hove Singularities (VHS) are occurred in this system and its position depends on twisted angle. This behaviour coming from the saddle points (SP) of band dispersion of TBG. The position of VHS will move away from Fermi level along with the increase of twisted angle. This result can be used to calculate others physical quantities such as optical conductivity or critical temperature of superconductivity that may occur in TBG.
\end{abstract}

Keywords - Density of States, Twisted Bilayer Graphene, Low Energy.

\section{INTRODUCTION}

Since first time isolated in 2004, Graphene has attracted a lot of interest from physicist to study its unique propeties such as ballistic electron transport, quantum Hall effect and chiral tunneling[1,2]. Recently, study in multilayer Graphene is more attractive since the electronic properties of Graphene can be modified by pilling up a few layers[3]. More interesting properties arise in Twisted Bilayer Graphene (TBG) system, in which two adjacent Graphene layers forms Bernal Stacking and rotated with respect to the others $[3,4,5]$. It has been known that van Hove Singularity (VHS) occured in TBG[6] which drives the speculation that superconductivity maybe manifest in this system[7,8]. For small rotation or twisted angle, $\theta \leq 10^{\circ}$, we can modify the position of VHS by tuning the commensurate rotation. This methods of tuning never seen in other materials $[4,5,6]$.

In this paper we report calculation of the Density of States (DOS) in TBG system. We assume that the TBG system has the same behaviour as the Pristine Graphene Monolayer (PGM), whereas the Fermi level located on Dirac Point. From this calculation we obtain logarithmic behaviour of DOS showing that VHS are occured in this system and its position depends on twisted angle.

\section{METHODS}

In order to obtain DOS, we calculate the magnitude of group velocity of Dirac Fermion. The group velocity can be calculated via divergence from the dispersion of energy $E(\vec{k})$ :

$$
\vec{v}=\frac{1}{\hbar} \nabla_{k} E(\vec{k})
$$

where $\hbar$ denotes Planck constant and $E(\vec{k})$ is given by [9]:

$$
E\left(k_{x}, k_{y}\right)=\alpha\left(A^{2}+B^{2}\right)^{1 / 2},
$$

where

$$
\begin{aligned}
& \alpha= \pm \frac{2 \hbar^{2} v_{f}^{2}}{15 t}, \\
& A=k_{x}^{2}-k_{y}^{2}-\frac{1}{4} \Delta K_{x}^{2}+\frac{1}{4} \Delta K_{y}^{2}, \\
& B=2 k_{x} k_{y}-\frac{1}{2} \Delta K_{x} \Delta K_{y},
\end{aligned}
$$

where $t$ is interlayer coupling and $\left(k_{x}, k_{y}\right)$ is two dimensional wave vector relative to the midpoint of the two Dirac Points[9]. The vector $\Delta K\left(\Delta K_{x}, \Delta K_{y}\right)$ is relative shift of the Dirac Points in the Brillouin Zone (BZ) (see Fig. 1) with[4,5]

$$
\Delta K=\frac{8 \pi}{3 a_{o}} \sin \left(\frac{\theta}{2}\right)
$$

where $a_{0}$ is a lattice constant of the Graphene monolayer $(\approx 2,46 \AA), \quad \theta$ is the angle of commensurate rotation, $\Delta K_{x}=\Delta K \sin (\theta / 2)$ and $\Delta K_{y}=\Delta K \cos (\theta / 2)[4,5,7]$.

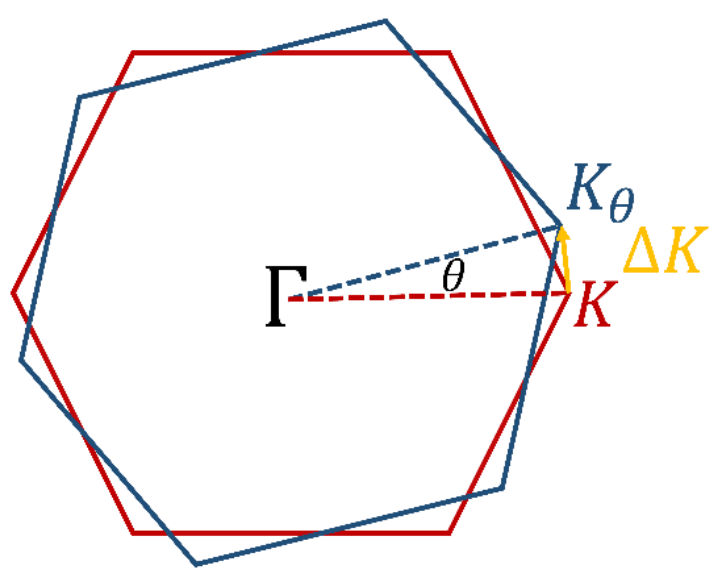

Figure 1. BZ of TBG 
DOS can be calculated via (4) below :

$$
D(E)=\frac{1}{4 \pi^{3} N \hbar} \int \frac{d l}{|\vec{v}|}
$$

where $d l$ is the element of length in the surface of energy and $N$ is a number of unit cell over volume or surface area.

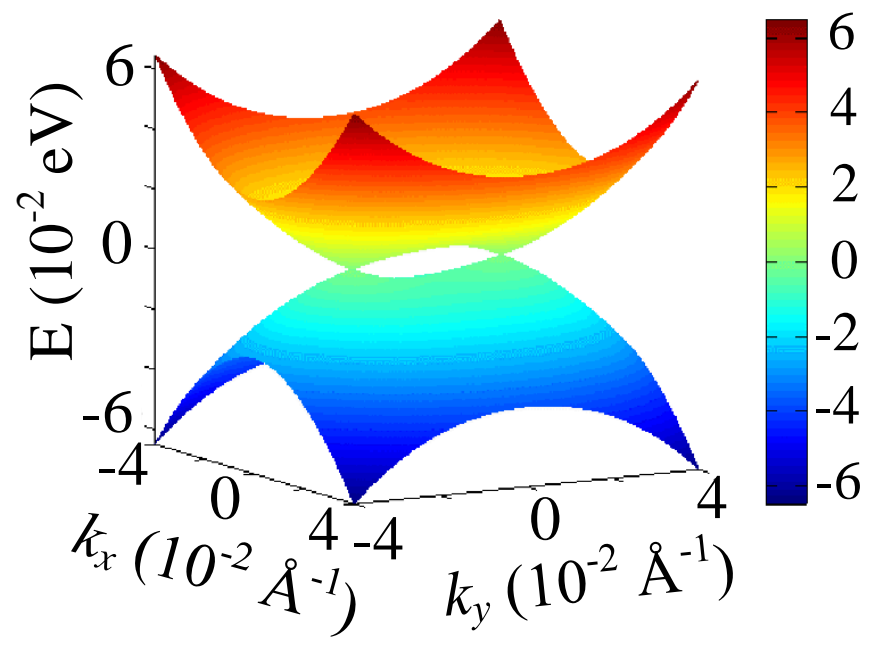

Figure 2. DE of TBG for the angle of commensurate rotation $1.16^{\circ}$.

\section{RESULTS}

The dispersion energy (DE) of TBG in (2) showing two split Dirac Cones with two VHS in the middle (see Fig. 2). Fig. 2 shows DE for the angle $1.16^{\circ}$. Fig. $3 \mathrm{a}$ and $3 \mathrm{~b}$ show saddle points (SP) in the valence band and conduction band respectively, which is the cause of VHS occurance. We change the scaling factor $(\alpha)$ in (2) from 2 becomes 99.24 to match VHS with the data of experiments $(6 \mathrm{meV}$ from Dirac Points) [6].

From (1) and (2), one can obtain the magnitude of group velocity which is given by:

$$
|\vec{v}|=\frac{2 \alpha \sqrt{k_{x}^{2}+k_{y}^{2}}}{\hbar} .
$$

Using (4) and (5), one can derive DOS as follow

$$
\begin{aligned}
& D(E)=\frac{1}{16 \alpha \pi^{3} N}\left(\ln \left|\frac{\beta \alpha^{2}+E_{f}^{2}}{\beta \alpha^{2}+E^{2}}\right|\right), \\
& \quad \beta=-2\left(A_{1}\left(k_{x}^{2}-k_{y}^{2}\right)+B_{1}\left(2 k_{x} k_{y}\right)\right)-\frac{1}{16}|\Delta K|^{4},
\end{aligned}
$$

where

$$
A_{1}=-\frac{1}{4} \Delta K_{x}^{2}+\frac{1}{4} \Delta K_{y}^{2}, \quad B_{1}=-\frac{1}{2} \Delta K_{x} \Delta K_{y} .
$$
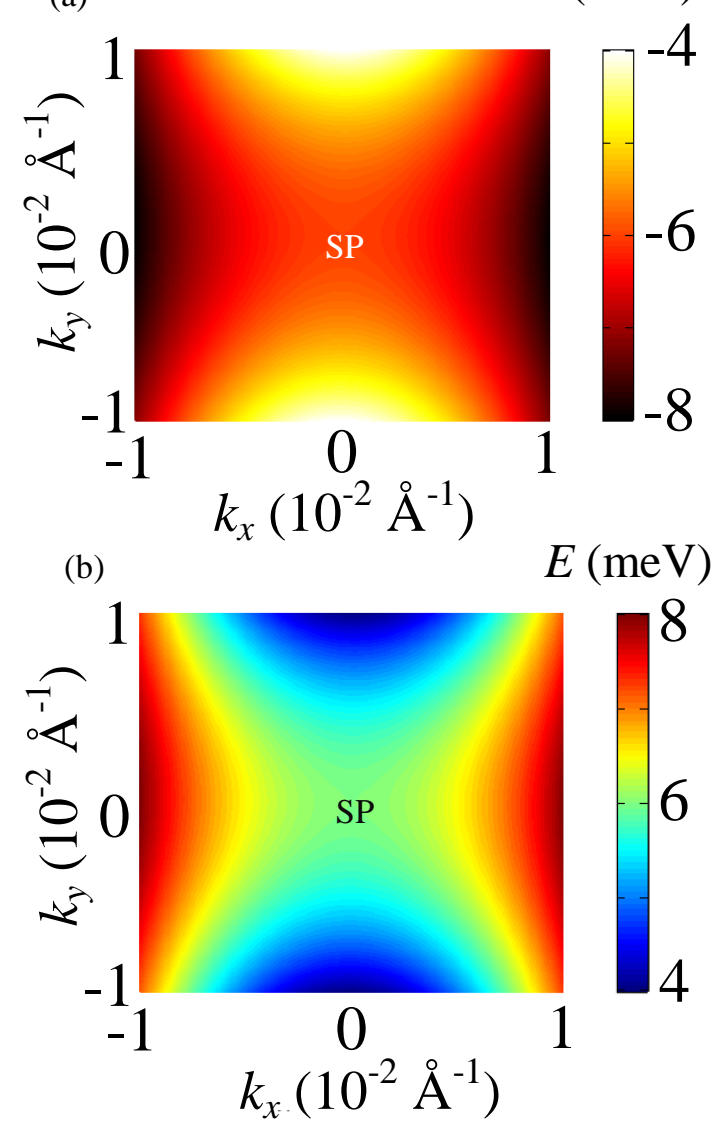

Figure 3. Saddle Points (SP) in the valence band (a) and conduction band (b) for the angle of rotation $1.16^{\circ}$.

In this calculation we assume that TBG model has the same behaviour as PGM in which the Fermi Level is located at the Dirac Points. Fig. 4 shows the plot of DOS derive from (6). In this figure, VHS is present indicated by the logarithmic shape of DOS at particular energy in the valence band and the conduction band. For the rotation angle of $1.79^{\circ}$, we change the scaling factor to 239.2 correspond to the VHS position about $41 \mathrm{meV}$ from Dirac Points. Similiar to rotation angle of $3.48^{\circ}$ the scaling factor set to 436.6 where, as a consequence, VHS is placed on 215 $\mathrm{meV}$ from Dirac Points[6]. In general, commensurate rotation angle tune the position of VHS 

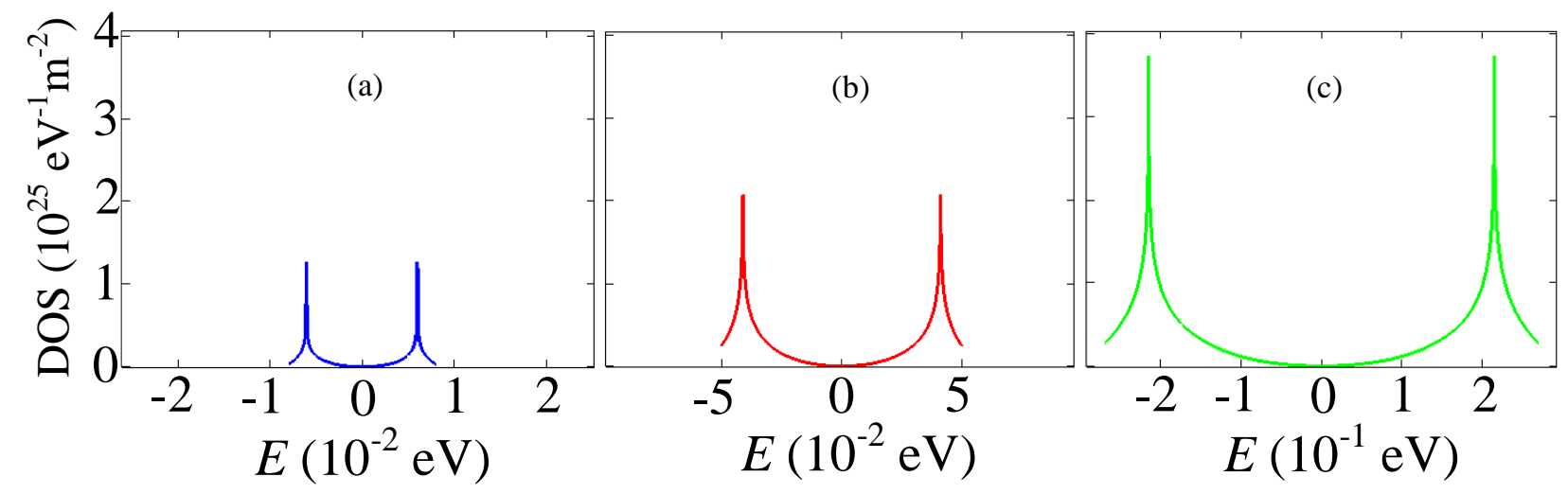

Figure 4. Plot of DOS for the angle of rotation (a) $1.16^{\circ}$ (b) $1.79^{\circ}$ and (c) $3.48^{\circ}$.

\section{CONCLUSION}

In conclusion we have reported the calculation of DOS for TBG system. We have shown that DOS of TBG has logarithmic behaviour known as VHS in the valence band and conduction band, respectively. These two peaks are related to the occurance of SP in DOS.

\section{ACKNOWLEDGMENT}

We would like to express our gratitude to Universitas Gadjah Mada in supporting our reseach.

\section{REFERENCES}

[1] A.H. Castro Neto, F. Guinea, N.M.R. Peres, K.S. Novoselov and A.K. Geim, "The Electronic Properties of Graphene," Rev.Mod.Phys., vol. 81, Jan. 2009, pp. 109-162, doi: 10.1103/RevModPhys.81.109.

[2] K.S. Novoselov, A.K. Geim, S.V. Morozov, D. Jiang, Y. Zhang, S.V. Dubonos, I.V. Grigorieva and A.A. Firsov, "Electric Field Effect in Atomically Thin Carbon Films," Science, vol. 306, Oct. 2004, pp 666-669, doi: 10.1126/science.1102896.

[3] E.S. Morel, P. Vargas, L. Chico and L. Brey, "Charge Redistribution and Interlayer Coupling in Twisted Bilayer Graphene Under Electric Fields," Phys.Rev.B, vol. 84, Nov. 2011, pp. 195421, doi: 10.1103/PhysRevB.84.195421.

[4] J.M.B. Lopes dos Santos, N.M.R. Peres and A.H. Castro Neto, "Graphene Bilayer with a Twist: Electronic Structure", Phys.Rev.Lett., vol. 99, Dec. 2007, pp. 256802, doi: 10.1103/PhysRevLett.99.256802.

[5] J.M.B. Lopes dos Santos, N.M.R. Peres and A.H. Castro Neto, "Continuum Model of The Twisted Graphene Bilayer," Phys.Rev.B, vol. 86, Oct. 2012, pp. 155449, doi: 10.1103/PhysRevB.86.155449.

[6] G. Li, A. Luican, J.M.B. Lopes dos Santos, A.H. Castro Neto, A. Reina, J. Kong and E.Y. Andrei, "Observation of Van Hove Singularities in Twisted Graphene Layers," Nature Phys., vol. 6, Feb. 2010, pp. 109-113, doi: 10.1038/NPHYS1463.
[7] M.N. Manaf, "Possibility of Superconductivity Instabilities in Twisted Graphene Layers," Master Tesis, Universitas Gadjah Mada, Yogyakarta, Indonesia, unpublished.

[8] J.L. McChesney, A. Bostwick, T. Ohta, T. Seyller, K. Horn, J. Gonzalez and E. Rotenberg, "Extended van Hove Singularity and Superconducting Instability in Doped Graphene," Phys.Rev.Lett., vol 104, Apr. 2010, pp. 136803, doi: 10.1103/PhysRevLett.104.136803.

[9] W. He, Z. Chu and L. He, "Chiral Tunneling in a Twisted Graphene Bilayer," Phys.Rev.Lett., vol. 111, Aug. 2013, pp. 066803, doi: 10.1103/PhysRevLett.111.066803. 